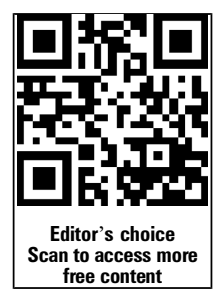

\title{
The effect of comedication with conventional synthetic disease modifying antirheumatic drugs on TNF inhibitor drug survival in patients with ankylosing spondylitis and undifferentiated spondyloarthritis: results from a nationwide prospective study
}

\author{
Elisabeth Lie, ${ }^{1,2}$ Lars Erik Kristensen, ${ }^{3,4}$ Helena Forsblad-d'Elia, ${ }^{1}$ \\ Tatiana Zverkova-Sandström, ${ }^{1}$ Johan Askling ${ }^{5}$ Lennart T Jacobsson, ${ }^{1}$ for the ARTIS \\ Study Group
}

\begin{abstract}
Handling editor Hans WJ Bijlsma

- Additional material is published online only. To view please visit the journal online (http://dx.doi.org/10.1136/ annrheumdis-2014-206616).

For numbered affiliations see end of article.
\end{abstract}

\section{Correspondence to}

Dr Elisabeth Lie, Department of Rheumatology, Diakonhjemmet Hospital, P.O. Box 23 Vinderen, Oslo N-0319, Norway;

elisabeth_lie@yahoo.no

Received 13 September 2014 Revised 1 February 2015

Accepted 2 February 2015 Published Online First 20 February 2015

CrossMark

\section{SLinked}

http://dx.doi.org/10.1136 annrheumdis-2014-206758

\section{ABSTRACT}

Objective To assess the effect of comedication with conventional synthetic disease modifying antirheumatic drugs (csDMARDs) on retention to tumour necrosis factor inhibitor (TNFi) therapy in patients with ankylosing spondylitis (AS) and undifferentiated spondyloarthritis (uSpA).

Methods Data on patients with a clinical diagnosis of AS or USpA starting treatment with adalimumab, etanercept or infliximab as their first TNFi during 20032010 were retrieved from the Swedish national biologics register and linked to national population based registers. Five-year drug survival was analysed by Cox regression with age, sex, baseline csDMARD comedication, TNFi type, prescription year and covariates representing frailty and socioeconomic status. AS and uSpA were analysed separately. Sensitivity analyses included models with csDMARD as a time-dependent covariate and adjustments for additional potential confounders.

Results 1365 patients with AS and 1155 patients with uSpA were included, of whom $40.8 \%$ versus $50.3 \%$ used csDMARD comedication at baseline. In the unadjusted analyses superior drug survival was observed for patients using versus not using csDMARD comedication among patients with $A S(p<0.001)$ but not among patients with uSpA ( $p=0.175)$. In the multivariable Cox regression analyses comedication with csDMARD was associated with better retention to TNFi therapy both in AS (HR $0.71, p<0.001$ ) and uSpA (HR 0.82, $p=0.020$ ). The results were similar with csDMARD comedication as a time-dependent covariate, and the associations were retained when adjusting for erythrocyte sedimentation rate, C-reactive protein, patient global, swollen joints, uveitis, psoriasis and inflammatory bowel disease. Conclusions In this large register study of patients with AS and USpA, use of csDMARD comedication was associated with better 5 -year retention to the first TNFi.

\section{INTRODUCTION}

Spondyloarthritis (SpA) is a group of related diseases associated with HLA-B27 and characterised by inflammation of the axial skeleton (causing inflammatory back pain), enthesitis, arthritis (most often monoarthritis or asymmetrical oligoarthritis affecting the lower limbs), and association to psoriasis, inflammatory bowel disease (IBD) and anterior uveitis. ${ }^{1}$ Ankylosing spondylitis (AS) is the classical SpA subtype and the subtype that has been best characterised. SpA can be classified as either axial or peripheral. ${ }^{2}{ }^{3}$

For rheumatoid arthritis (RA) it has consistently been shown that comedication with methotrexate (MTX) increases the clinical efficacy of tumour necrosis factor inhibitor (TNFi) treatment and reduces structural joint damage. ${ }^{4}$ MTX is also a frequently used treatment for psoriatic arthritis (PsA), but no randomised controlled trials have addressed whether comedication with conventional synthetic disease modifying drug (csDMARD) increases the efficacy of TNFi in this group. On the other hand, studies based on treatment register data have shown a better survival on drug of TNFi with MTX comedication in PsA. ${ }^{5-7}$

One possible mechanism for lack/loss of efficacy with TNFi treatment is formation of antidrug antibodies, and studies in AS have shown that formation of such antibodies was associated with clinical non-response to infliximab (IFX) and adalimumab $(\mathrm{ADA})^{8-10}$ while the role of antidrug antibodies against etanercept (ETN) is less clear. ${ }^{11}$ Based on the experience with csDMARD/immunomodulator comedication in other diseases such as RA and Crohn's disease, a general effect of these drugs on immunogenicity of anti-TNF monoclonal antibodies has been suggested. ${ }^{12-14}$

The current Assessment of SpondyloArthritis International Society/European League Against Rheumatism (ASAS/EULAR) recommendations for the management of AS state that there is no evidence to support the obligatory use of concomitant DMARD with anti-TNF therapy in patients with axial disease. ${ }^{15}$ This recommendation reflects that there is no clear evidence for a positive effect of comedication with MTX or other csDMARDs on 
efficacy or drug survival of TNFi in AS/axial SpA, but this is not an issue that has been much studied. One randomised trial comparing standard continuous and on-demand IFX in AS partly addressed the questions of MTX comedication by randomly assigning the patients in the on-demand group to treatment with $(n=62)$ and without $(n=61)$ MTX for 58 weeks. No statistically significant effects of MTX could be demonstrated, but a trend towards a lower rate of infusion reactions and somewhat higher response rates was observed, and the trial was not powered to assess the effect of comedication. ${ }^{16} \mathrm{~A}$ few other smaller studies have also been published, with conflicting results. ${ }^{17-19}$ Unlike similar analyses in PsA, MTX has not been shown to be a predictor of prolonged drug survival in AS. ${ }^{20-22}$

The primary objective of the current study was to assess if comedication with csDMARDs is associated with TNFi drug survival in patients with AS and undifferentiated SpA (uSpA). Second, we wanted to explore whether such an association, if present, would be consistent across different TNFis and reasons for discontinuation, as well as to explore which other factors influence TNFi drug survival in this patient group.

\section{METHODS}

\section{Data source and patients}

Data for this study were retrieved from the Swedish Biologics Register (ARTIS) which was established in 1999 and described in detail elsewhere. ${ }^{23}$ The register is overseen by the Swedish Rheumatology Association and is integrated into clinical practice. Disease activity and treatment is registered at initiation of biological DMARD treatment and at regular follow-up visits by the treating rheumatologist. For this study, patients with a diagnosis of AS (International Classification of Diseases (ICD)-10 code M45) or uSpA (ICD-10 code M46.8) in ARTIS starting their first treatment course with a TNFi, which could be either ADA, ETN or IFX, between 1 January 2003 and 31 December 2010 were included. Patients with a diagnosis of psoriatic arthritis (ICD-10 L40.5) were not included, neither were patients starting treatment with golimumab and certolizumab pegol $(\mathrm{N}=18)$ during the same period. Follow-up data from ARTIS were available through 31 December 2011.

For the subgroup of patients starting TNFi from 1 January 2006 onwards we could use data from the Swedish Prescribed Drugs Register (PDR) to define four groups of patients: (1) patients starting csDMARD around the time of TNFi start ('starters'), (2) patients already using csDMARD during the 6-month period before TNFi start and continuing csDMARD after TNFi start ('continued users'), (3) patients using csDMARD during the 6-month period before TNFi start and stopping csDMARD when starting TNFi ('stoppers') and (4) patients not using csDMARD during the 6-month period before TNFi start and not starting csDMARD with TNFi ('non-users'). Further details on the PDR, methods and definitions are included as online supplementary material.

\section{Covariates}

Data on age, sex, use of csDMARD comedication, any previous csDMARD treatment, disease duration and disease activity variables (erythrocyte sedimentation rate (ESR) $(\mathrm{mm} / \mathrm{h})$, C-reactive protein (CRP) (mg/L), patient global $100-\mathrm{mm}$ visual analogue scale (VAS), 28-swollen joint count (28-SJC)) were extracted from ARTIS.

Information on the presence of the $\mathrm{SpA}$ related comorbidities uveitis, psoriasis or IBD prior to start of TNFi treatment was based on data from the Swedish National Patient Register (NPR), requiring at least one registered visit with an ICD-code for the respective diagnosis. The NPR is kept by the National Board of Health and Welfare, it was started in 1964, obtained complete national coverage for inpatient care in 1987, and has included specialised outpatient care since 2001 (http:// www.socialstyrelsen.se/register/helsodataregister/patientregistret/ inenglish). The NPR also provided the number of hospital days and non-primary outpatient care visits due to any cause during the 2-year period prior to start of TNFi. These variables were used as measures of general patient frailty. ${ }^{24}$

To adjust for socioeconomic status we used data on educational level and disposable income provided by Statistics Sweden. Income data were available for the period 2002-2008, and we thus used 2008 income data for patients started on TNFi in 2010 and income data from the year prior to TNFi start for all other patients. Income data were recalculated to $1000 €$ per year.

\section{Outcome}

The main outcome was 5-year drug survival. Stop date was defined as the date of TNFi discontinuation as registered in ARTIS. Patients discontinuing TNFi due to either remission/ inactive disease or pregnancy were censored at the date of discontinuation and thus not counted as events in the survival analyses. Patients without a stop date were censored at the minimum of death date, date of latest visit +450 days, or 31 December 2011. The reason for discontinuation was recorded in ARTIS.

\section{Statistical analyses}

Baseline data are presented as frequencies with percentages, means with SDs and/or medians with 25th, 75th percentiles depending on the type of data and their distribution. Baseline characteristics were compared between patients using and not using csDMARD comedication at baseline (start of TNFi) using $\chi^{2}$ test, independent samples $t$ test and Mann-Whitney U test, as appropriate. For analysis of drug survival we used KaplanMeier analysis with log-rank test comparing patients with and without csDMARD comedication. However, the primary analysis was a multivariable Cox regression analysis of 5-year drug survival including the following covariates, selected a priori: age, sex, csDMARD comedication at baseline (yes vs no), TNFi type, start year (2003-2006 vs 2007-2010), hospital days and number of outpatient visits during the 2 years prior to TNFi start, and disposable income and level of education. HRs with 95\% CIs are presented. We also performed separate KaplanMeier analyses comparing patients with and without csDMARD comedication for each of the three TNFi included in the study, and separate analyses for each of the main reasons for discontinuation, that is, primary lack of efficacy, secondary lack (loss) of efficacy, lack of efficacy (both primary and secondary) and safety, with censoring for other the reasons for discontinuation.

\section{Sensitivity analyses}

Univariable Cox regression analyses were performed for the variables included in the multivariable model as well as for baseline ESR, CRP, patient global assessment, 28-SJC and presence of joint swelling (28-SJC $\geq 1$ ), and the presence of uveitis, IBD and psoriasis. Variables not included in the primary multivariable Cox regression models were then one by one included in the multivariable models to check for confounding in relation to the effect of csDMARD comedication. For several of these variables there was a considerable amount of missing data (up to $33 \%$ ) and these variables were thus not included in the primary analyses. As additional sensitivity analyses we performed 
multivariable Cox regression models with csDMARD comedication as a time-dependent covariate and with csDMARD comedication at baseline grouped as 'MTX vs other vs none' instead of 'yes vs no'.

We also applied the multivariable Cox regression model from the primary analysis to the pooled group of AS and $\mathrm{uSpA}$, excluding patients with swollen joints at baseline. Furthermore, in patients starting TNFi from 1 January 2006 onwards we did multivariable Cox regression analysis including the csDMARD groups 'starters', 'continued users', 'stoppers' and 'non-users', as defined above-in AS, uSpA, and the pooled group of AS and uSpA (again excluding patients with swollen joints at baseline).

Statistical tests were two-sided, and p values below 0.05 were considered statistically significant. IBM SPSS Statistics V.21 and SAS V.9.3 were used for the statistical analyses.

\section{RESULTS}

\section{Patients and baseline characteristics}

In total, 1365 patients diagnosed with AS and 1155 patients with uSpA starting their first TNFi 2003-2010 were included. At start of TNFi treatment, csDMARD comedication was used by $40.8 \%$ of patients with AS and $50.3 \%$ of patients with uSpA (table 1). Such comedication was more often given with IFX (55.4\% and $60.7 \%$, respectively) than with ADA $(28.1 \%$ / $37.7 \%)$ and ETN (30.5\%/49.1\%). The majority $(77.0 \%$ and $79.2 \%$, respectively) of those on comedication used MTX. The $\mathrm{uSpA}$ group included higher proportions of female patients and comorbidity with IBD and psoriasis, and a lower proportion with prior uveitis compared with patients with AS. Table 1 also shows baseline characteristics for patients with and without csDMARD comedication. Patients who were on comedication had higher levels of ESR and CRP, more often had at least one swollen joint, and had a higher number of outpatient visits during the 2 years prior to TNFi start.

\section{Follow-up and discontinuations}

Overall, 631 (46.2\%) of patients with AS and 652 (56.4\%) with uSpA discontinued TNFi treatment during follow-up, and the majority (585 and 628, respectively) did so during the first 5 years. Median (25th, 75 th percentile) total follow-up time was $777(394.5,1481.5)$ versus $670(267,1391)$ days for AS and uSpA, respectively. Patients with AS and uSpA who discontinued TNFi stayed on treatment for $383(148,839)$ and 324.5 $(132.25,746)$ days, respectively. Lack of efficacy was the most frequent reason for discontinuation reported, followed by safety (table 2).

\section{Unadjusted drug survival analyses}

Drug survival was better for AS versus uSpA (figure 1A; estimated median overall survival was 5.3 vs 3.3 years, $\mathrm{p}<0.001$ ). There was a statistically significant difference in drug survival in favour of csDMARD comedication in the AS group (figure 1B; $\mathrm{p}<0.001$ ), but not in the uSpA group (figure $1 \mathrm{C} ; \mathrm{p}=0.175$ ). Survival curves for patients with and without csDMARD comedication are shown separately for ADA, ETN and IFX in online supplementary figure S1A-F. Among patients with AS drug survival was statistically significantly superior in patients using csDMARD comedication within all three TNFi (see online supplementary figure S1A-C), while differences were not statistically significant for patients with uSpA (see online supplementary figure S1D-F). Separate analyses of different reasons for discontinuation were somewhat limited by small numbers. In AS there was a statistically significant association between csDMARD comedication and discontinuations due to safety $(p=0.021)$ while the difference was not statistically significant for lack of efficacy $(p=0.077)$ (data not shown). Among patients with uSpA those with csDMARD comedication there was a trend towards an association between csDMARD comedication and discontinuations due to safety $(p=0.127)$ while there was no association with discontinuations due to lack of efficacy (data not shown).

\section{Multivariable Cox regression analysis}

Separate models for AS and $\mathrm{uSpA}$ are shown in table 3 . Adjusting for age, sex, TNFi type, start year (2007-2010 vs 2003-2006), number of hospital days and outpatient visits 2 years prior to inclusion, income and education, 5-year retention to therapy was superior for patients who received csDMARD comedication both in the AS group (HR (95\% CI) 0.71 (0.59 to $0.85), \mathrm{p}<0.001)$ and in the uSpA group $(0.82$ (0.68 to 0.97$)$, $\mathrm{p}=0.020)$. Sex, TNFi type, start year and hospital days were also statistically significantly associated with TNFi drug survival in both patient groups (table 3 ).

\section{Sensitivity analyses}

Results from univariable Cox regression analyses are shown in online supplementary table S1. Statistically significant associations were observed for disease activity measures like ESR, CRP, patient global VAS, and 28-SJC as well as for uveitis. Online supplementary tables S2A and B show the results of adding additional variables, representing disease activity and SpA-related comorbidities, to the multivariable models in table 3 , with particular attention to the effect of csDMARD comedication. In AS, all additional variables that were statistically significant by univariable analysis remained significant in the multivariable model, and, importantly, the effect of csDMARD comedication remained statistically significant at a $p$ value of $<0.001$ (see online supplementary table S2A). Estimates were virtually not changed by adding uveitis, while the effect of csDMARD comedication was somewhat enhanced when the covariates reflecting disease activity were added to the model. Similar findings were observed for the uSpA group (see online supplementary table S2B).

Among patients with AS 223 (16\%) changed csDMARD comedication during follow-up (158 patients discontinued and 65 patients started csDMARD comedication, respectively). The corresponding number among patients with SpA was 221 (19\%; 148 patients discontinuing and 73 patients starting csDMARD). Analysing csDMARD use as a time-dependent covariate did not substantially change the results, however, in the AS group the HR estimate for csDMARD comedication changed from 0.71 to 0.61 (see table 4 and online supplementary table S3).

To examine whether the effect of csDMARD comedication was due to MTX only or also due to other csDMARDs, we grouped csDMARD comedication as 'MTX' (including csDMARD combinations with MTX), 'other' and 'none' (no comedication), with the latter as the reference. For AS there was a statistically significant association with TNFi drug survival both for comedication with MTX (HR 0.74, p=0.004) and for comedication with other csDMARDs (HR 0.59, $\mathrm{p}=0.002$ ), whereas for uSpA the association was only observed for MTX (HR $0.79, \mathrm{p}=0.010$ ) (see table 4 and online supplementary table S4).

We also performed multivariable Cox regression on the pooled group of patients with AS and $\mathrm{uSpA}$, excluding patients with swollen joints (by 28-SJC) at baseline $(\mathrm{N}=1839$ ). Use of csDMARD at baseline was statistically significantly associated with 5-year drug survival with an HR of 0.76 (table 4). 
Table 1 Baseline demographics and disease characteristics

\begin{tabular}{|c|c|c|c|c|c|c|c|c|}
\hline & \multicolumn{4}{|l|}{ AS } & \multicolumn{4}{|l|}{ uSpA } \\
\hline & $\begin{array}{l}\text { All } \\
N=1365\end{array}$ & $\begin{array}{l}\text { CsDMARD } \\
\text { comedication } \\
\mathrm{N}=557\end{array}$ & $\begin{array}{l}\text { No csDMARD } \\
\text { comedication } \\
\mathrm{N}=808\end{array}$ & $p$ Value & $\begin{array}{l}\text { All } \\
N=1155\end{array}$ & $\begin{array}{l}\text { csDMARD } \\
\text { comedication } \\
\mathrm{N}=581\end{array}$ & $\begin{array}{l}\text { No csDMARD } \\
\text { comedication } \\
\mathrm{N}=574\end{array}$ & $\mathrm{p}$ Value \\
\hline Age, mean (SD) & $43.8(12.3)$ & $43.6(12.2)$ & $43.9(12.4)$ & 0.692 & $42.6(12.1)$ & $42.8(12.4)$ & $42.3(11.9)$ & 0.520 \\
\hline Sex, n (\%) male & $995(72.9)$ & 410 (73.6) & $585(72.4)$ & 0.622 & $606(52.5)$ & $293(50.4)$ & $313(54.5)$ & 0.163 \\
\hline TNFi type & & & & $<0.001$ & & & & $<0.001$ \\
\hline Adalimumab, n (\%) & $406(29.7)$ & $114(20.5)$ & $292(36.1)$ & & $326(28.2)$ & $123(21.2)$ & $203(35.4)$ & \\
\hline Etanercept, $\mathrm{n}(\%)$ & $354(25.9)$ & $108(19.4)$ & $246(30.4)$ & & $391(33.9)$ & $192(33.0)$ & $199(34.7)$ & \\
\hline Infliximab, n (\%) & $605(44.3)$ & $335(60.1)$ & $270(33.4)$ & & 438 (37.9) & $266(45.8)$ & $172(30.0)$ & \\
\hline Year of TNFi start & & & & $<0.001$ & & & & $<0.001$ \\
\hline 2003-2006, n (\%) & 459 (33.6) & $254(45.6)$ & $205(25.4)$ & & 460 (39.8) & $274(47.2)$ & $186(32.4)$ & \\
\hline 2007-2010, n (\%) & $906(66.4)$ & $303(54.4)$ & $603(74.6)$ & & $695(60.2)$ & 307 (52.8) & $388(67.6)$ & \\
\hline cSDMARD comedication & & & & NA & & & & NA \\
\hline MTX, n (\%) & $389(28.5)$ & & & & $401(34.7)$ & & & \\
\hline SSZ, n (\%) & $108(7.9)$ & & & & $100(8.7)$ & & & \\
\hline Other, $\mathrm{n}(\%)^{*}$ & $60(4.4)$ & & & & $80(6.9)$ & & & \\
\hline None, $\mathrm{n}(\%)$ & $808(59.2)$ & & & & $574(49.7)$ & & & \\
\hline MTX dose, mg, mean (SD)†‡ & $\begin{array}{l}13.9(4.9) \\
(\mathrm{N}=424) \ddagger\end{array}$ & & & & $\begin{array}{l}15.8(5.3) \\
(\mathrm{N}=456) \ddagger\end{array}$ & & & \\
\hline Disease duration (years), median $(25,75$ percentile $) \dagger$ & $\begin{array}{l}14.0(6.7,24.7) \\
(\mathrm{N}=1327)\end{array}$ & $\begin{array}{l}13.4(6.7,24.1) \\
(\mathrm{N}=537)\end{array}$ & $14.6(6.4,25.0)(\mathrm{N}=790)$ & 0.514 & $\begin{array}{l}8.7(3.2,17.5) \\
(\mathrm{N}=1135)\end{array}$ & $\begin{array}{l}7.9(3.0,16.3) \\
(\mathrm{N}=571)\end{array}$ & $9.5(3.5,18.9)(\mathrm{N}=564)$ & 0.079 \\
\hline $\begin{array}{l}\text { Number of previous csDMARDs, mean (SD)/median } \\
\text { (25th, } 75 \text { th percentile) }\end{array}$ & $\begin{array}{l}0.40(0.69) \\
0(0,1)\end{array}$ & $\begin{array}{l}0.54(0.78) \\
0(0,1)\end{array}$ & $\begin{array}{l}0.30(0.60) \\
0(0,0)\end{array}$ & $<0.001$ & $\begin{array}{l}0.43(0.84) \\
0(0,1)\end{array}$ & $\begin{array}{l}0.49(0.86) \\
0(0,1)\end{array}$ & $\begin{array}{l}0.38(0.82) \\
0(0,0)\end{array}$ & 0.001 \\
\hline ESR (mm/h), median (25th, 75th percentile)† & $\begin{array}{l}20(10,37) \\
(\mathrm{N}=1104)\end{array}$ & $\begin{array}{l}24(10.25,40.75) \\
(\mathrm{N}=496)\end{array}$ & $18(9,34)(\mathrm{N}=608)$ & 0.001 & $18(8,36)(\mathrm{N}=989)$ & $20(10,41)(N=540)$ & $16(8,33.5)(\mathrm{N}=449)$ & 0.003 \\
\hline CRP (mg/L), median (25th, 75th percentile) $\dagger$ & $13(5,30)(N=1055)$ & $17(7,36)(N=485)$ & $12(5,25)(N=570)$ & $<0.001$ & $\begin{array}{l}11(4,27.5) \\
(\mathrm{N}=929)\end{array}$ & $12(5,35)(N=519)$ & $9(3,21)(N=410)$ & $<0.001$ \\
\hline Patient global VAS, mean (SD)† & $57.5(24.0)(\mathrm{N}=925)$ & $57.3(23.7)(\mathrm{N}=433)$ & $59.0(24.3)(\mathrm{N}=492)$ & 0.784 & $61.0(21.7)(\mathrm{N}=924)$ & $60.8(21.9)(\mathrm{N}=507)$ & $61.3(21.5)(\mathrm{N}=417)$ & 0.731 \\
\hline 28-swollen joint count $\geq 1, n(\%) \dagger$ & $260(28.8)(N=904)$ & $159(37.3)(\mathrm{N}=426)$ & $101(21.1)(\mathrm{N}=478)$ & $<0.001$ & $421(46.1)(\mathrm{N}=913)$ & $270(53.0)(N=509)$ & $151(37.4)(\mathrm{N}=404)$ & $<0.001$ \\
\hline $\begin{array}{l}\text { 28-swollen joint count, mean (SD)/median (25th, 75th } \\
\text { percentile) } \dagger\end{array}$ & $\begin{array}{l}1.1(2.6) \\
0(0,1)(N=904)\end{array}$ & $\begin{array}{l}1.4(2.9) \\
0(0,2) \\
(\mathrm{N}=426)\end{array}$ & $\begin{array}{l}0.8(2.2) \\
0(0,0) \\
(\mathrm{N}=478)\end{array}$ & $<0.001$ & $\begin{array}{l}1.8(3.1) \\
0(0,2) \\
(\mathrm{N}=913)\end{array}$ & $\begin{array}{l}2.2(3.4) \\
1(0,3)(N=509)\end{array}$ & $\begin{array}{l}1.3(2.6) \\
0(0,2)(N=404)\end{array}$ & $<0.001$ \\
\hline Uveitis, n (\%) & $361(26.4)$ & $153(27.5)$ & $208(25.7)$ & 0.477 & $205(17.7)$ & $93(16.0)$ & $112(19.5)$ & 0.119 \\
\hline Inflammatory bowel disease, $\mathrm{n}(\%)$ & $120(8.8)$ & $57(10.2)$ & $63(7.8)$ & 0.118 & $120(10.4)$ & $54(9.3)$ & $66(11.5)$ & 0.220 \\
\hline Psoriasis, $n(\%)$ & $67(4.9)$ & $29(5.2)$ & $38(4.7)$ & 0.672 & $116(10.0)$ & $61(10.5)$ & $55(9.6)$ & 0.604 \\
\hline $\begin{array}{l}\text { Number of hospital days, mean (SD)/median (25th, 75th } \\
\text { percentile)§ }\end{array}$ & $\begin{array}{l}3.6(12.2) \\
0(0,1)\end{array}$ & $\begin{array}{l}3.4(10.6) \\
0(0,1)\end{array}$ & $\begin{array}{l}3.8(13.2) \\
0(0,1)\end{array}$ & 0.663 & $\begin{array}{l}5.0(17.4) \\
0(0,3)\end{array}$ & $\begin{array}{l}5.7(21.8) \\
0(0,2.5)\end{array}$ & $\begin{array}{l}4.3(11.1) \\
0(0,3)\end{array}$ & 0.641 \\
\hline $\begin{array}{l}\text { Number of outpatient visits, mean (SD) / median (25th, } \\
\text { 75th percentile)§ }\end{array}$ & $\begin{array}{l}7.6(6.7) \\
6(3,10)\end{array}$ & $\begin{array}{l}8.0(6.4) \\
6(3,11)\end{array}$ & $\begin{array}{l}7.3(6.9) \\
5(3,10)\end{array}$ & 0.008 & $\begin{array}{l}10.6(9.2) \\
8(5,14)\end{array}$ & $\begin{array}{l}11.3(9.4) \\
9(5,15)\end{array}$ & $\begin{array}{l}9.8(9.0) \\
7(4,13)\end{array}$ & 0.001 \\
\hline Disposable income (in $1000 €$ ), mean (SD) & $\begin{array}{l}22.0(16.0) \\
(\mathrm{N}=1342)\end{array}$ & $21.0(13.8)(\mathrm{N}=552)$ & $22.7(17.3)(\mathrm{N}=790)$ & 0.066 & $\begin{array}{l}22.0(21.5) \\
(\mathrm{N}=1149)\end{array}$ & $21.0(14.3)(\mathrm{N}=577)$ & $23.0(26.8)(N=572)$ & 0.102 \\
\hline
\end{tabular}




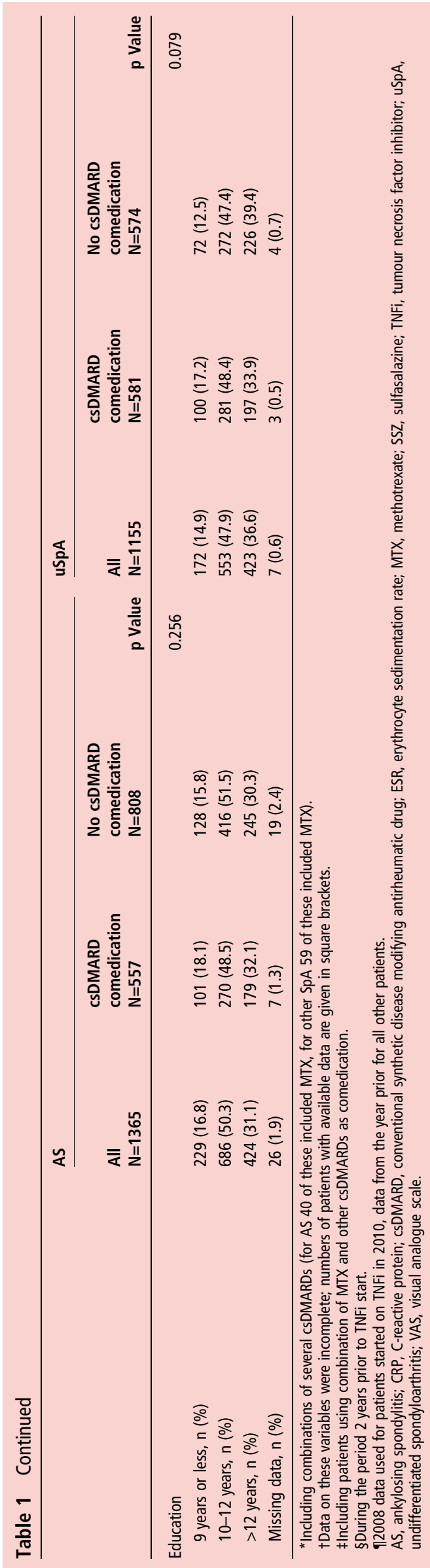

Table 2 Reasons for discontinuation

\begin{tabular}{|c|c|c|c|c|}
\hline & \multicolumn{2}{|c|}{ AS (total $\mathrm{N}=1365$ ) } & \multicolumn{2}{|c|}{ uSpA (total $\mathrm{N}=1155$ ) } \\
\hline & All & $\begin{array}{l}\text { First } 5 \\
\text { years }\end{array}$ & All & $\begin{array}{l}\text { First } 5 \\
\text { years }\end{array}$ \\
\hline Lack of efficacy & $234(37.1 \%)$ & $213(36.4 \%)$ & $272(41.7 \%)$ & $260(41.4 \%)$ \\
\hline Primary LOE & $114(18.1 \%)$ & $113(19.3 \%)$ & $129(19.8 \%)$ & $129(20.5 \%)$ \\
\hline Secondary LOE & 120 (19.0\%) & 100 (17.1\%) & $143(21.9 \%)$ & $131(20.9 \%)$ \\
\hline Safety & $183(29.0 \%)$ & $178(30.4 \%)$ & $161(24.7 \%)$ & $157(25.0 \%)$ \\
\hline $\begin{array}{l}\text { Remission/inactive } \\
\text { disease* }^{*}\end{array}$ & $26(4.1 \%)$ & $25(4.3 \%)$ & $35(5.4 \%)$ & $35(5.6 \%)$ \\
\hline Pregnancy* & $11(1.7 \%)$ & $9(1.5 \%)$ & $16(2.5 \%)$ & $16(2.5 \%)$ \\
\hline Other reasons & $146(23.1 \%)$ & $135(23.1 \%)$ & $111(17.0 \%)$ & $107(17.0 \%)$ \\
\hline Reason missing & $31(4.9 \%)$ & $25(4.3 \%)$ & $57(8.7 \%)$ & $53(8.4 \%)$ \\
\hline Total & 631 & 585 & 652 & 628 \\
\hline
\end{tabular}

For the final sensitivity analyses, including patients starting TNFi after 1 January 2006 and using medication data from the PDR, we identified 108 'csDMARD starters' with AS and 90 with uSpA. In AS, this group had better drug survival than 'csDMARD non-users' (HR 0.72, $\mathrm{p}=0.058$ ), but did not do quite as well as 'continued users' and 'stoppers' (table 4). In uSpA, however, 'starters' did just as well as 'continued users' and better than both 'stoppers' and 'non-users' (HR 0.71, $\mathrm{p}=0.058$ ). In the pooled group of patients with AS and $\mathrm{uSpA}$ (excluding those with swollen joints at baseline) the difference between 'starters' $(n=168)$ and 'non-users' $(n=666)$ was accentuated (HR 0.65, p=0.002; table 4).

\section{DISCUSSION}

While there has been some indication that csDMARDs comedication with TNFi is of no additional value in AS, ${ }^{16}{ }^{18-21}$ this is not a question that has been extensively studied. Keeping in mind that the effect of comedication is of unequivocal importance in RA and that there also is some evidence supporting a benefit in PsA, ${ }^{5} 7$ we set out to study this question in AS and uSpA. When adjusting for potential confounders we found a statistically significant beneficial effect of csDMARD comedication on TNFi drug survival both in AS and in uSpA. The effect was more pronounced in AS (HR 0.71) compared with uSpA (HR 0.82), and present both for MTX and other csDMARDs in AS, while in the uSpA group we could only find an effect for MTX. Due to missing data we chose to adjust for measures of disease activity in separate models (table 3), in which the association with csDMARD comedication remained stable or tended to be strengthened.

Although the available data are limited, the efficacy of csDMARDs in axial SpA is considered doubtful or modest, and in the ASAS/EULAR treatment recommendations for the management of AS they are not recommended for patients with pure axial disease. ${ }^{15}$ However, csDMARDs have been included as a treatment option for patients with coexisting peripheral disease. $^{15}$

The finding of an association between csDMARD comedication and TNFi retention in AS and uSpA in our study might be due to several mechanisms, among which are prevention of antidrug antibody formation, a separate anti-inflammatory effect of csDMARDs, as well as residual confounding. Since these data 
A

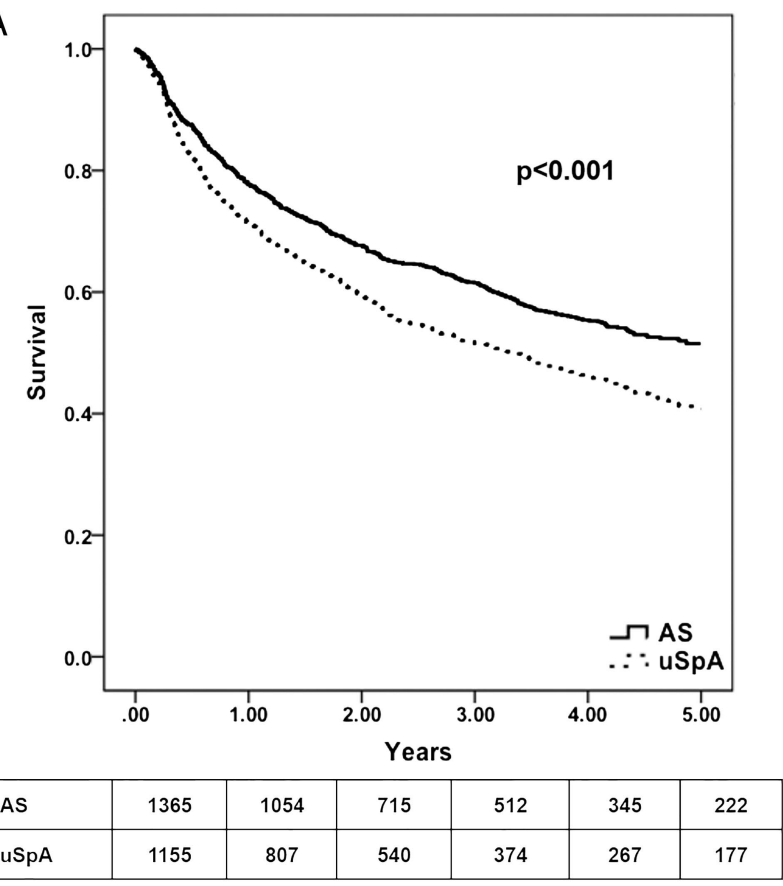

B

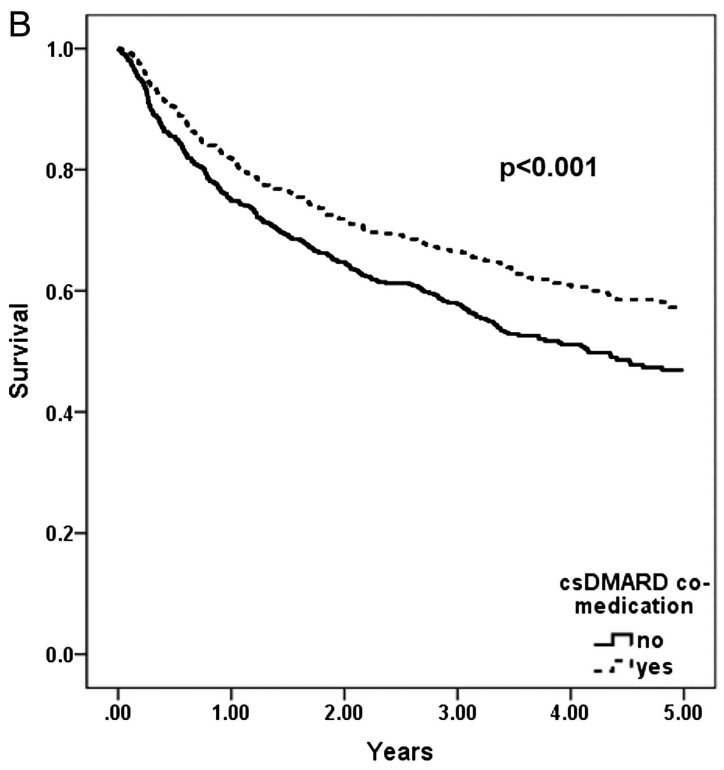

\begin{tabular}{|l|l|l|l|l|l|l|}
\hline Co-med. & 557 & 454 & 329 & 263 & 186 & 131 \\
\hline No co-med. & 808 & 600 & 386 & 249 & 159 & 91 \\
\hline
\end{tabular}

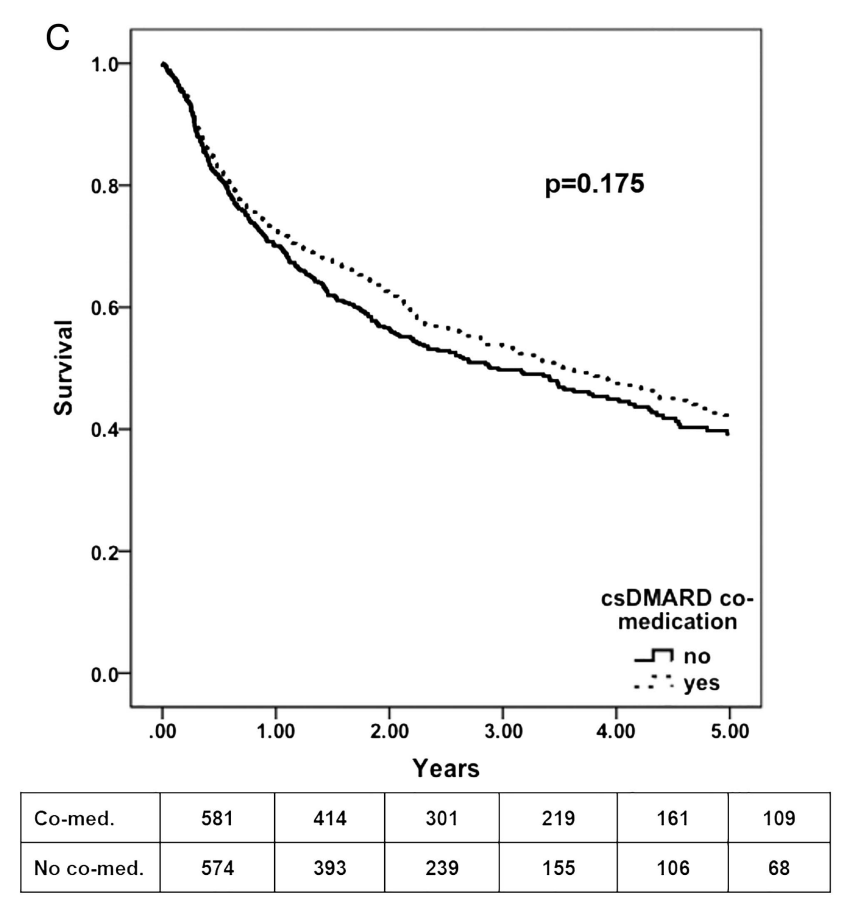

Figure 1 Kaplan-Meier curves of 5-year drug survival of the first prescribed TNFi. (A) AS versus uSpA. (B) AS csDMARD comedication versus no csDMARD comedication. (C) uSpA csDMARD comedication versus no csDMARD comedication. $p$ Values are based on log-rank test. The tables show the number of patients at risk at baseline, 1, 2, 3, 4 and 5 years in each group. AS, ankylosing spondylitis; cSDMARD, conventional synthetic disease modifying anti-rheumatic drug; TNFi, tumour necrosis factor inhibitor; uSpA, undifferentiated spondyloarthritis.

were observational, the allocation to treatment-both use of csDMARD comedication and choice of TNFi-was far from random, and changed over time. Although we were able to perform adjustments for several potential confounders, residual confounding is still likely to be present, as is the case for all observational studies. As expected, csDMARD comedication was more often used in patients with (co-occurrence of) peripheral arthritis (table 1), but adjusting for this did not change the HR estimates for csDMARD comedication, and a similar statistically significant association was observed in the pooled group of patients with AS and USpA without swollen joints (table 4). In current clinical practice, a very relevant question is whether a csDMARD should be started together with the TNFi in a patient with axial SpA without active peripheral disease. Due to a heterogeneous patient population, including many patients with prior csDMARD exposure, and missing information on some relevant confounders, we could only partly address this question in our study. Our sensitivity analyses to address the issue did indicate benefit of csDMARD co-therapy initiated with the TNFi (table 4), but among patients with AS, this subgroup 
Table 3 Multivariable Cox regression analysis of 5-year survival of the first TNFi in AS and uSpA

\begin{tabular}{|c|c|c|c|c|c|c|c|c|}
\hline & \multicolumn{4}{|l|}{ AS } & \multicolumn{4}{|l|}{ uSpA } \\
\hline & B & SE & $p$ Value & HR $(95 \% \mathrm{Cl})$ & B & SE & $\mathrm{p}$ Value & HR (95\% Cl) \\
\hline Age (per 10 years) & 0.012 & 0.036 & 0.739 & 1.01 (0.94 to 1.09$)$ & 0.021 & 0.035 & 0.552 & 1.02 (0.95 to 1.09$)$ \\
\hline Sex (ref. female) & -0.412 & 0.094 & $<0.001$ & 0.66 (0.55 to 0.80$)$ & -0.381 & 0.088 & $<0.001$ & 0.68 (0.57 to 0.81$)$ \\
\hline csDMARD comedication (ref. none) & -0.349 & 0.094 & $<0.001$ & 0.71 (0.59 to 0.85 ) & -0.201 & 0.086 & 0.020 & $0.82(0.69$ to 0.97$)$ \\
\hline TNFi type & & & 0.037 & & & & $<0.001$ & \\
\hline ADA vs IFX & -0.107 & 0.107 & 0.317 & 0.90 (0.73 to 1.11$)$ & -0.365 & 0.111 & 0.001 & 0.69 (0.56 to 0.86$)$ \\
\hline ETN vs IFX & -0.288 & 0.112 & 0.010 & 0.75 (0.60 to 0.93 ) & -0.339 & 0.099 & 0.001 & 0.71 (0.59 to 0.86$)$ \\
\hline Start year $2007-2010$ vs $2003-2006$ & 0.203 & 0.097 & 0.037 & 1.22 (1.01 to 1.48$)$ & 0.259 & 0.092 & 0.005 & $1.30(1.08$ to 1.55$)$ \\
\hline Number of hospital days* & 0.008 & 0.003 & 0.008 & 1.01 (1.00 to 1.01$)$ & 0.005 & 0.002 & 0.006 & $1.00(1.00$ to 1.01$)$ \\
\hline Number of outpatient visits* & 0.016 & 0.006 & 0.010 & $1.02(1.00$ to 1.03$)$ & 0.005 & 0.005 & 0.264 & $1.01(1.00$ to 1.01$)$ \\
\hline Disposable income (per $1000 €) \dagger$ & -0.005 & 0.004 & 0.146 & $0.99(0.99$ to 1.00$)$ & -0.002 & 0.003 & 0.459 & $1.00(0.99$ to 1.00$)$ \\
\hline Education & & & 0.087 & & & & 0.032 & \\
\hline $10-12$ years vs $\leq 9$ years & -0.075 & 0.119 & 0.532 & 0.93 (0.73 to 1.17$)$ & -0.131 & 0.120 & 0.275 & $0.88(0.69$ to 1.11$)$ \\
\hline$>12$ years vs $\leq 9$ years & -0.253 & 0.134 & 0.060 & 0.78 (0.60 to 1.01$)$ & -0.355 & 0.131 & 0.007 & 0.70 (0.54 to 0.91$)$ \\
\hline Missing vs $\leq 9$ years & 0.937 & 0.719 & 0.192 & 2.55 (0.62 to 10.4$)$ & -10.29 & 102.1 & 0.920 & $0.00(0.00$ to $\infty)$ \\
\hline
\end{tabular}

* During the period 2 years prior to TNFi start.

†2008 data used for patients started on TNFi in 2010, data from the year prior for all other patients.

ADA, adalimumab; AS, ankylosing spondylitis; CSDMARD, conventional synthetic disease modifying antirheumatic drug; ETN, etanercept; IFX, infliximab; TNFi, tumour necrosis factor inhibitor; uSpA, undifferentiated spondyloarthritis.

did not do as well as the group stopping csDMARD when starting TNFi.

In RA MTX co-therapy is believed to have effects beyond prevention of formation of antidrug antibodies. ${ }^{4}$ Conversely, in an observational study in PsA no evidence of such a 'synergistic' effect was found, as response rates of TNFi were similar with and without MTX, while there was an effect on drug survival for IFX, a trend for ADA, but no effect for ETN. ${ }^{7}$ Since formation of antidrug antibodies is more a feature of treatment with IFX or ADA than with ETN, this might suggest an effect of MTX on such antibody formation. In the current study, we did not find a similar 'gradient' regarding the effect of comedication across the three TNFi. We also tested the interaction term 'csDMARD comedication $\times$ TNFi type' in the multivariable Cox regression models, and it was not statistically significant (data not shown; $p$ value 0.63 for AS and 0.82 for $u S p A$ ).

There are several strengths of the present study. The study is large and national with minimal selection bias since over $85 \%$ of initiated courses with TNFi are included according to recent estimates. We were able to adjust for several possible confounders including socioeconomic factors and frailty through linkage to population-based registers, which are independent data sources. Furthermore, we were able to also analyse csDMARD comedication as a time-dependent covariate, and the results

Table 4 Sensitivity analyses: estimates for csDMARD comedication from multivariable Cox regression models

\begin{tabular}{|c|c|c|c|c|c|c|}
\hline & \multicolumn{2}{|l|}{ AS } & \multicolumn{2}{|l|}{ uSpA } & \multicolumn{2}{|c|}{ Pooled group AS and uSpA } \\
\hline & HR $(95 \% \mathrm{Cl})$ & $\mathrm{p}$ Value & HR $(95 \% \mathrm{Cl})$ & $\mathrm{p}$ Value & HR $(95 \%$ Cl) & $\mathrm{p}$ Value \\
\hline Main analyses (see table 3 for full models AS and uSpA) & 0.71 (0.59 to 0.85$)$ & $<0.001$ & $0.82(0.69$ to 0.97$)$ & 0.020 & & \\
\hline Time-varying csDMARD covariate & 0.61 (0.51 to 0.74 ) & $<0.001$ & 0.81 (0.69 to 0.96$)$ & 0.017 & & \\
\hline Alternative csDMARD categories & & 0.001 & & 0.034 & & \\
\hline MTX vs none & 0.74 (0.61 to 0.91$)$ & 0.004 & 0.79 (0.66 to 0.94$)$ & 0.010 & & \\
\hline Other vs none & $0.59(0.41$ to 0.83$)$ & 0.002 & $0.94(0.71$ to 1.26$)$ & 0.691 & & \\
\hline Exclusion of patients with swollen joints at baseline* & & & & & 0.76 (0.66 to 0.89$)$ & $<0.001$ \\
\hline csDMARD groups $†$ & & $<0.001$ & & 0.017 & & \\
\hline csDMARD starters vs non-users & $0.72(0.52$ to 1.01$)$ & 0.058 & 0.71 (0.49 to 1.01$)$ & 0.058 & & \\
\hline csDMARD continued users vs non-users & 0.50 (0.38 to 0.64$)$ & $<0.001$ & $0.75(0.59$ to 0.95$)$ & 0.018 & & \\
\hline csDMARD stoppers vs non-users & 0.65 (0.48 to 0.89$)$ & 0.006 & $1.05(0.80$ to 1.39$)$ & 0.716 & & \\
\hline csDMARD groups $\ddagger$ & & & & & & $<0.001$ \\
\hline csDMARD starters vs non-users & & & & & $0.65(0.50$ to 0.85$)$ & 0.002 \\
\hline csDMARD continued users vs non-users & & & & & $0.62(0.51$ to 0.76$)$ & $<0.001$ \\
\hline csDMARD stoppers vs non-users & & & & & $0.79(0.63$ to 1.00$)$ & 0.049 \\
\hline
\end{tabular}


were consistent with those obtained when applying csDMARD comedication at baseline.

Some limitations should also be acknowledged. We did not have sufficient data on disease activity measures at baseline and follow-up to study the association between csDMARD comedication and clinical response. Thus drug survival was used as a surrogate measure for effectiveness. Misclassification of AS and uSpA could be a problem. We have, however, recently validated and found good validity for the diagnoses of AS and SpA in large patient samples of randomly selected clinical records in the NPR. ${ }^{25}$ The patients in the present study, who were treated with TNFi, constitute subgroups with more severe disease where proportions fulfilling classification criteria would be expected to be higher. ${ }^{26}$ Based on this it is a likely assumption that the majority of the AS patients in the current study fulfilled the 1984 Modified New York criteria for AS. The uSpA group, on the other hand, did according to our validation study probably mostly consist of patients with non-radiographic axial $\mathrm{SpA},{ }^{2}$ with a smaller proportion with peripheral $\mathrm{SpA} .^{3}$ The $\mathrm{uSpA}$ group included a higher proportion of female patients than the AS group, which is in accordance with published epidemiological data. ${ }^{27}$ The proportions with uveitis, IBD and psoriasis in our study are also in line with recently published prevalence data, ${ }^{28}$ supporting the validity of the diagnoses, but the lack of data on HLA-B27 and imaging is a limitation. The Bath Ankylosing Spondylitis Disease Activity Index was included in ARTIS only at the end of the study period and was therefore only available for a minority of patients.

In conclusion, we found that patients with AS and uSpA who received csDMARD comedication with their first TNFi remained on therapy significantly longer than those who were not on comedication. The association remained statistically significant when adjusting for an array of potential confounders, however, the mechanisms behind it remain to be elucidated. Furthermore, the beneficial effect of co-therapy with csDMARDs, if present, may not be large enough to justify changes in management recommendations.

\author{
Author affiliations \\ ${ }^{1}$ Department of Rheumatology and Inflammation Research, Sahlgrenska Academy at \\ University of Gothenburg, Gothenburg, Sweden \\ ${ }^{2}$ Department of Rheumatology, Diakonhjemmet Hospital, Oslo, Norway \\ ${ }^{3}$ Section of Rheumatology, Department of Clinical Sciences, Lund University, Malmö, \\ Sweden \\ ${ }^{4}$ Musculoskeletal Statistics Unit, Department of Rheumatology, The Parker Institute, \\ Copenhagen University Hospital, Bispebjerg and Frederiksberg, Copenhagen, \\ Denmark \\ ${ }^{5}$ Clinical Epidemiology Unit \& Rheumatology Unit, Department of Medicine, \\ Karolinska Institutet, Stockholm, Sweden
}

Collaborators The ARTIS Study Group: Eva Baecklund, Nils Feltelius, Alf Kastbom, Lars Klareskog, Solbritt Rantapää-Dahlqvist, Ronald van Vollenhoven.

Contributors Study concept and design: EL, LTJ. Acquisition of data: LEK, HFdE, JA, LTJ, the ARTIS group. Statistical analyses: EL, TZ. Drafting of the manuscript: EL, LTJ. Critical revision of the manuscript for important intellectual content: EL, LEK, HE, TZ, JA, LTJ.

Funding The ARTIS Study Group conducts scientific analyses using data from the Swedish Biologics Register (ARTIS) run by the Swedish Society for Rheumatology. For the maintenance of this register, the Swedish Society for Rheumatology has received funding, independent of the conduct of these scientific analyses, from Merck, BMS, Pfizer, AbbVie, SOBI, UCB, Astra Zeneca and Roche. This specific study further received funding from the Swedish Foundation for Strategic Research.

Competing interests EL: Consulting and/or speaker honoraria from AbbVie, Bristol-Myers Squibb, Hospira, Pfizer and UCB. LEK: Consulting and/or speaker honoraria from AbbVie, MSD, Pfizer and UCB. HF: No competing interests. TZ: No competing interests. JA: Consulting and/or speaker honoraria from AbbVie, Bristol-Myers Squibb, Pfizer, Roche, UCB and MSD. LTJ: Consulting honoraria from AbbVie, Pfizer and UCB.
Ethics approval Ethical approval was granted by the Regional Ethics Committee, Karolinska Institutet, Stockholm, Sweden (ethical approval number: 2011/29-31/1).

Provenance and peer review Not commissioned; externally peer reviewed.

Data sharing statement Additional data and information about the study can be accessed by contacting the corresponding author.

\section{REFERENCES}

1 Dougados M, Baeten D. Spondyloarthritis. Lancet 2011;377:2127-37.

2 Rudwaleit M, van der Heijde D, Landewe R, et al. The development of Assessment of SpondyloArthritis international Society classification criteria for axial spondyloarthritis (part II): validation and final selection. Ann Rheum Dis 2009;68:777-83.

3 Rudwaleit M, van der Heijde D, Landewe R, et al. The Assessment of SpondyloArthritis International Society classification criteria for peripheral spondyloarthritis and for spondyloarthritis in general. Ann Rheum Dis 2011;70:25-31

4 Nam JL, Winthrop KL, van Vollenhoven RF, et al. Current evidence for the management of rheumatoid arthritis with biological disease-modifying antirheumatic drugs: a systematic literature review informing the EULAR recommendations for the management of RA. Ann Rheum Dis 2010;69:976-86.

5 Glintborg B, Ostergaard M, Dreyer L, et al. Treatment response, drug survival, and predictors thereof in 764 patients with psoriatic arthritis treated with anti-tumor necrosis factor alpha therapy: results from the nationwide Danish DANBIO registry. Arthritis Rheum 2011;63:382-90.

6 Kristensen LE, Gulfe A, Saxne T, et al. Efficacy and tolerability of anti-tumour necrosis factor therapy in psoriatic arthritis patients: results from the South Swedish Arthritis Treatment Group register. Ann Rheum Dis 2008;67:364-9.

7 Fagerli $\mathrm{KM}$, Lie $\mathrm{E}$, van der Heijde $\mathrm{D}$, et al. The role of methotrexate co-medication in TNF-inhibitor treatment in patients with psoriatic arthritis: results from 440 patients included in the NOR-DMARD study. Ann Rheum Dis 2014;73:132-7

8 de Vries MK, Wolbink GJ, Stapel SO, et al. Inefficacy of infliximab in ankylosing spondylitis is correlated with antibody formation. Ann Rheum Dis 2007;66:133-4.

9 de Vries MK, Brouwer E, van der Horst-Bruinsma IE, et al. Decreased clinical response to adalimumab in ankylosing spondylitis is associated with antibody formation. Ann Rheum Dis 2009;68:1787-8.

10 Plasencia C, Pascual-Salcedo D, Nuno L, et al. Influence of immunogenicity on the efficacy of longterm treatment of spondyloarthritis with infliximab. Ann Rheum Dis 2012:71:1955-60.

11 de Vries MK, van der Horst-Bruinsma IE, Nurmohamed MT, et al. Immunogenicity does not influence treatment with etanercept in patients with ankylosing spondylitis. Ann Rheum Dis 2009;68:531-5.

12 Krieckaert $\mathrm{CL}$, Bartelds GM, Lems WF, et al. The effect of immunomodulators on the immunogenicity of TNF-blocking therapeutic monoclonal antibodies: a review. Arthritis Res Ther 2010;12:217.

13 Jani M, Barton A, Warren RB, et al. The role of DMARDs in reducing the immunogenicity of TNF inhibitors in chronic inflammatory diseases. Rheumatology (Oxford) 2014;53:213-22.

14 Garces S, Demengeot J, Benito-Garcia E. The immunogenicity of anti-TNF therapy in immune-mediated inflammatory diseases: a systematic review of the literature with a meta-analysis. Ann Rheum Dis 2013;72:1947-55.

15 Braun J, van den Berg R, Baraliakos X, et al. 2010 update of the ASAS/EULAR recommendations for the management of ankylosing spondylitis. Ann Rheum Dis 2011;70:896-904.

16 Breban M, Ravaud $\mathrm{P}$, Claudepierre $\mathrm{P}$, et al. Maintenance of infliximab treatment in ankylosing spondylitis: results of a one-year randomized controlled trial comparing systematic versus on-demand treatment. Arthritis Rheum 2008;58:88-97.

17 Perez-Guijo VC, Cravo AR, Castro Mdel C, et al. Increased efficacy of infliximab associated with methotrexate in ankylosing spondylitis. Joint Bone Spine 2007;74:254-8

18 Mulleman D, Lauferon F, Wendling D, et al. Infliximab in ankylosing spondylitis: alone or in combination with methotrexate? A pharmacokinetic comparative study. Arthritis Res Ther 2011;13:R82.

19 Ternant D, Mulleman D, Lauferon F, et al. Influence of methotrexate on infliximab pharmacokinetics and pharmacodynamics in ankylosing spondylitis. $\mathrm{Br} / \mathrm{Clin}$ Pharmacol 2012;73:55-65.

20 Glintborg B, Ostergaard M, Krogh NS, et al. Predictors of treatment response and drug continuation in 842 patients with ankylosing spondylitis treated with anti-tumour necrosis factor: results from 8 years' surveillance in the Danish nationwide DANBIO registry. Ann Rheum Dis 2010;69:2002-8.

21 Heiberg MS, Koldingsnes W, Mikkelsen K, et al. The comparative one-year performance of anti-tumor necrosis factor alpha drugs in patients with rheumatoid arthritis, psoriatic arthritis, and ankylosing spondylitis: results from a longitudinal, observational, multicenter study. Arthritis Rheum 2008;59:234-40.

22 Kristensen LE, Karlsson JA, Englund M, et al. Presence of peripheral arthritis and male sex predicting continuation of anti-tumor necrosis factor therapy in ankylosing 


\section{Clinical and epidemiological research}

spondylitis: an observational prospective cohort study from the South Swedish Arthritis Treatment Group Register. Arthritis Care Res (Hoboken) 2010;62:1362-9.

23 Askling J, Fored CM, Geborek P, et al. Swedish registers to examine drug safety and clinical issues in RA. Ann Rheum Dis 2006;65:707-12.

24 Neovius $M$, Arkema EV, Olsson $\mathrm{H}$, et al. Drug survival on TNF inhibitors in patients with rheumatoid arthritis comparison of adalimumab, etanercept and infliximab. Ann Rheum Dis 2015;74:354-60.

25 Lindström U, Exarchou S, Sigurdardottir V, et al. Validity of ankylosing spondylitis and spondyloarthritis diagnoses in the Swedish national patient register [abstract]. Ann Rheum Dis 2014;73(Suppl2):SAT0098.
26 Kristensen LE, Petersson IF, Geborek $\mathrm{P}$, et al. Sick leave in patients with ankylosing spondylitis before and after anti-TNF therapy: a population-based cohort study. Rheumatology (Oxford) 2012;51:243-9.

27 Tournadre A, Pereira B, Lhoste A, et al. Differences between women and men with recent-onset axial spondyloarthritis: results from a prospective multicenter French cohort. Arthritis Care Res (Hoboken) 2013;65:1482-9.

28 Stolwijk C, Essers I, van Tubergen A, et al. The epidemiology of extra-articular manifestations in ankylosing spondylitis: a population-based matched cohort study. Ann Rheum Dis 2014. Published Online First 25 Mar 2014. doi:10.1136/ annrheumdis-2014-205253 